\title{
Cracking behaviour of PVD tungsten coatings deposited on steel substrates
}

\author{
Thomas Ganne ${ }^{\mathrm{ab}}$, Jérôme Crépin ${ }^{\mathrm{b}}$, Serge Serror ${ }^{\mathrm{a}}$, André Zaoui ${ }^{\mathrm{b}, *}$ \\ a Centre Technique d'Arcueil, 16 Bis avenue Prieur de la Côte d'Or, F-94114 Arcueil Cedex, France

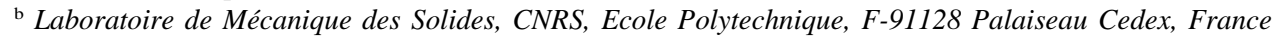

\begin{abstract}
Tungsten coatings have been deposited on steel substrates by magnetron sputtering. For the same processing con-ditions, the increase of the coating thickness enhances the (111) component of the crystallographic texture whereas the residual stress level decreases. Tensile and four-point bending tests, associated with an acoustic emission analysis, have been performed inside a SEM chamber in order to study the cracking mechanisms. When the residual stresses are taken into account, an intrinsic critical cracking stress and the associated energy release rate can be determined; the obtained values suggest an intergranular cracking mechanism. No debonding has been observed at the interface despite the large plastic deformation of the substrate at the crack tips. The observed strain localisation modes in the substrate near the interface have been shown to have a major influence on the limit crack density
\end{abstract}

Keywords: Magnetron sputtering; Residual stresses; Crystallographic texture; Critical cracking stress; Tungsten; Coating

\section{Introduction}

Coatings are more and more frequently used in order to improve the mechanical, thermal and chemical properties of various substrates. Their shielding effect has been proved to be highly efficient as long as they are not subjected to significant mechanical damage. Consequently, any optimisation procedure of the coating-substrate systems requires the identification of the damage

* Corresponding author. Tel.: +33-1-69333370; fax: +33-169333026.

E-mail address: zaoui@1ms.polytechnique.fr (A. Zaoui). mechanisms and a good understanding of the connection between microstructural characteristics, which strongly depend on the processing conditions [1], and the global damage behaviour [2]. This paper aims at improving the understanding and the quantitative description of the damage behaviour of brittle PVD coatings deposited on a ductile substrate from the experimental study of tungsten-steel systems.

This analysis cannot be achieved without an accurate characterisation of the initial state of the coatings, especially their residual stresses and crystallographic texture. Thermal stresses are the result of cooling from the deposition to room temperature, due to the thermal expansion coefficient mis- 
match between the coating and the substrate. Additional residual stresses, the so-called "intrinsic stresses", result from growth mechanisms: they are due to the accumulation of crystallographic flaws developing in the coating [1]. They are generally compressive, with an order of magnitude of 1 to 4 GPa. A number of studies have been devoted to the investigation of residual stresses in such coatings but the influence of the processing conditions and of the coating thickness on their overall level still needs to be known more precisely; the same conclusion applies to crystallographic textures. These questions are investigated in W-steel systems in Section 3.

The damage mechanisms which are the most frequently active in such systems are the coating mode I cracking and the interface debonding [2$3]$. The quantitative mechanical characterisation of damage initiation and kinetics requires adequate experimental techniques: in this study, in-situ tensile and four-point bending tests have been performed inside a S.E.M. chamber; an acoustic emission analysis has been developed for the study of the cracking kinetics whereas a microextensometry technique has been used for a better investigation of the strain field heterogeneity along the interface. The combined use of these techniques has led to the identification of the active cracking mechanisms, and to the determination of an intrinsic critical cracking stress and of the associated energy release rate from Hu-Evans's model [4] (Section 4). Moreover a preliminary analysis of the cracking kinetics has been conducted (Section 5), through the comparison of the experimentally defined minimum distance between two neighbour cracks with the one which can be predicted from Hu-Evans's model [4]; special attention has been paid to the influence of the coating thickness and partial results have been obtained on the influence of the processing conditions and the substrate yield stress and plastic behaviour on the limit crack density.

\section{Materials}

Tungsten coatings have been deposited on steel substrates by magnetron sputtering. The technical details of the sputtering procedure used have been reported in a previous paper [5]. Two processing conditions have been chosen. The first ones, called "A conditions" (3 kW target power, 3.5 Pa Ar pressure, $-25 \mathrm{~V}$ substrate bias; samples labelled from A to I with a coating thickness ranging from 0.6 to $30 \mu \mathrm{m}$ ), have been used to study the influence of the coating thickness on the overall mechanical properties of the samples (residual stresses, critical cracking stress, ...). The second processing conditions, called "B conditions" $(1.5 \mathrm{~kW}$ target power, $2 \mathrm{~Pa}$ Ar pressure, $-25 \mathrm{~V}$ substrate bias; samples $J$ and $K$ ) have been chosen for $30 \mu \mathrm{m}$ thick coatings in order to check the dependence of the cracking mechanisms on the processing parameters. In both cases, the obtained coatings exhibit a specific microstructure of densely packed fibrous grains.

The experimental analysis of the mechanical characteristics of the coatings, such as the residual stresses or the critical cracking stress, requires the knowledge of their elastic properties. Several authors [6-8] have determined the Young modulus of PVD tungsten coatings submitted to a compressive residual stresses state by nanoindentation and bending tests: they have shown that the experimental values, ranging from 360 to $430 \mathrm{GPa}$, are close to those of the bulk material (410 GPa). So, we assume in what follows that the Young modulus, the Poisson ratio as well as the coefficient of thermal expansion of our coatings, which are submitted to compressive residual stresses, are given by the bulk tungsten values (see Table 1).

Most of the studied substrates, constituted of a 35 NCDV12 steel, exhibit a martensitic structure. Some of them ("type 2") have been subjected to an annealing treatment in order to modify their yield stress so as to be able to investigate the influence of the substrate yield stress on the cracking behaviour of the coatings. The mechanical properties of both substrates are also reported in Table 1. The different sample characteristics (coating thickness, processing conditions and substrate) are reported in Table 2.

\section{Crystallographic texture, residual stresses and chemical analysis}

The initial state of the coatings studied has been characterised through the investigation of their 
Table 1

Mechanical properties and thermal expansion coefficients of PVD tungsten coatings and 35NCDV12 steel substrates

\begin{tabular}{lllll}
\hline & $E(\mathrm{GPa})$ & $v$ & $\alpha\left(10^{-6} \mathrm{~K}^{-1}\right)$ & Yield stress, $\sigma_{\mathrm{y}}(\mathrm{MPa})$ \\
\hline PVD W & 410 & 0.28 & 4.6 & - \\
Substrate type 1 & 200 & 0.29 & 12.3 & 1100 \\
Substrate type 2 & 200 & 0.29 & 12.3 & 800 \\
\hline
\end{tabular}

Table 2

Sample characteristics

\begin{tabular}{llll}
\hline Sample & Coating Thickness $(\mu \mathrm{m})$ & Elaboration conditions & Substrate \\
\hline A & 0.6 & A & Type 1 \\
B & 1.6 & A & Type 1 \\
C & 1.6 & A & Type 1 \\
D & 6 & A & Type 1 \\
E & 13.9 & A & Type 1 \\
F & 13.9 & A & Type 2 \\
G & 31.1 & A & Type 1 \\
H & 30.9 & A & Type 1 \\
I & 30.1 & A & Type 2 \\
J & 30.4 & B & Type 1 \\
K & 30.2 & B & Type 2 \\
\hline
\end{tabular}

crystallographic texture, residual stress state and chemical composition.

\subsection{Crystallographic texture}

The crystallographic texture of f.c.c coatings with a $\langle 110\rangle$ growth orientation [9] and of b.c.c coatings with a $\langle 111\rangle$ growth orientation [10] has already been shown to depend on their thickness. This dependence has been checked on our samples through an X-ray diffraction analysis using a $\mathrm{Cu}$ $\mathrm{K}_{\alpha}$ radiation; the analysed volume is $1 \mathrm{~mm}^{2} \times 1 \mu \mathrm{m}$ (average depth) large. The texture has been characterised by the texture coefficient $T_{\mathrm{hkl}}$ [11] which is defined, for any (hkl) orientation, as the ratio of the diffraction ray intensity, $I_{\mathrm{hkl}}$, to the theoretical diffraction ray intensity of an isotropic tungsten powder, $I_{\mathrm{o}, \mathrm{hkl}}$, normalised by the average value of these ratios over the number $n$ of (hkl) rays studied:

$T_{\mathrm{hk} l}=\left(\frac{I_{\mathrm{hkl}}}{I_{\mathrm{o}, \mathrm{hkl}}}\right) /\left(n^{-1} \sum_{i=1}^{n} \frac{I_{\mathrm{hkl}}^{i}}{I_{\mathrm{o}, \mathrm{hkl}}^{i}}\right)$ where $I_{\mathrm{hkl}}=(\mathrm{hkl})$ studied ray intensity, $I_{\mathrm{o}, \mathrm{hkl}}=(\mathrm{hkl})$ theoretical ray intensity, and $n=$ number of (hkl) rays studied. The greater this coefficient with respect to 1 , the stronger the texture along the considered direction. This analysis has been confirmed by classical pole figures. Figure 1 shows that the $T_{222}$ coefficient increases with the coating thickness, which characterises the development of a $\langle 111\rangle$ texture. This result is in agreement with Gergaud's [10] observations on Mo coatings, which

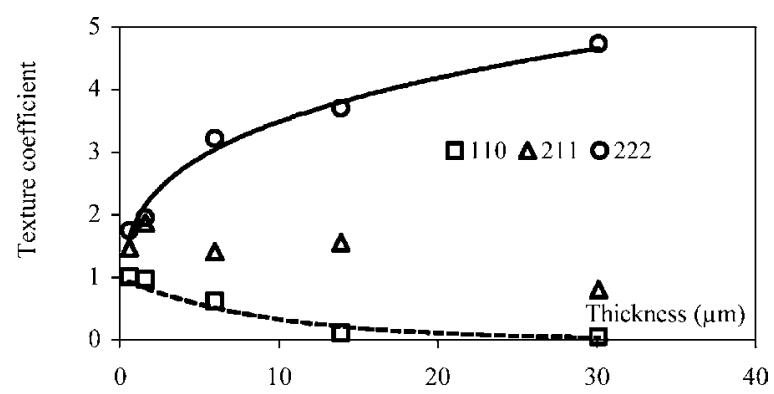

Fig. 1. Variation of the coating texture coefficient versus the thickness. 
have the same crystallographic structure. The texture can be considered as isotropic only for the 0.6 $\mu \mathrm{m}$ thick A coating.

\subsection{Residual stresses}

Residual stresses have been determined by Xray diffraction with a $\mathrm{Cu} \mathrm{K}$ radiation $\left(\lambda_{\mathrm{cu}}=0.1504\right.$ $\mathrm{nm})$, according to the " $\sin ^{2} \psi$ " method [12]. From the Bragg relation, the measured lattice spacing of $\{\mathrm{hkl}\}$ planes, $d_{\mathrm{hkl}}$, is linked to the elastic strain component $\varepsilon_{\phi \psi}$ by:

$$
\begin{aligned}
& \varepsilon_{\phi \psi}=\ln \left(\frac{d_{\mathrm{hkl}}}{d_{\mathrm{hkl}}^{0}}\right) \approx \frac{d_{\mathrm{hkl}}-d_{\mathrm{kh} 1}^{0}}{d_{\mathrm{hk} 1}^{0}}=-\operatorname{cotan}(\theta) \cdot(\theta \\
& \left.-\theta_{0}\right)
\end{aligned}
$$

where $d_{\mathrm{hkl}}^{0}$ is the stress-free lattice spacing and $\varepsilon_{\phi \psi}$ refers to the Euler angles $\phi$ and $\psi$ defined in Fig. 2 . The $\{123\}$ planes have been chosen for a better measurement accuracy.

Since tungsten single crystals can be considered as elastically isotropic [13], the same property is true for textured polycrystalline coatings [14]. According to a Reuss-type assumption (homogeneous stress state in the diffracted volume), the stress-strain relation is given by:

$$
\begin{aligned}
\varepsilon_{\phi \psi} & =\frac{1+v}{E}\left(\sigma_{11} \cos ^{2} \phi+\sigma_{12} \sin 2 \phi\right. \\
+ & \left.\sigma_{22} \sin ^{2} \phi-\sigma_{33}\right) \sin ^{2} \psi+\frac{1+v}{E} \sigma_{33}-\frac{v}{E}\left(\sigma_{11}\right.
\end{aligned}
$$

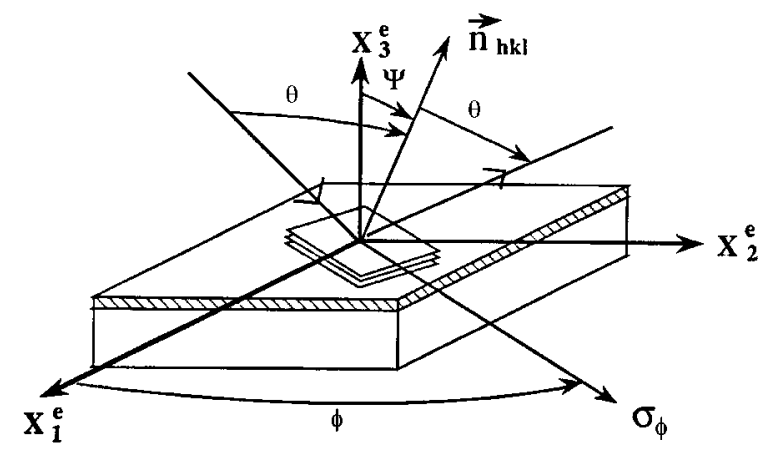

Fig. 2. Definition of $\psi, \phi$, and $\theta$ angles in the sample axes $\left(\mathrm{X}_{1}^{\mathrm{e}}, \mathrm{X}_{2}^{\mathrm{e}}, \mathrm{X}_{3}^{\mathrm{e}}\right)$.

$$
\begin{aligned}
& \left.+\sigma_{22}+\sigma_{33}\right)+\frac{1+v}{E}\left(\sigma_{13} \cos \phi\right. \\
& \left.+\sigma_{23} \sin \phi\right) \sin 2 \psi .
\end{aligned}
$$

In addition, if we assume an isotropic residual plane stress state $\left(\sigma_{i 3} \quad[i=\{1,2,3\}]=\sigma_{12}=0 \quad\right.$ and $\sigma_{11}=\sigma_{22}=\sigma$ ), eq. (3) is independent of $\phi$ :

$\varepsilon_{\phi \psi}=\frac{1+v}{E} \cdot \sigma \cdot \sin ^{2} \psi-\frac{v}{E} \cdot \sigma$,

so that $\sigma$ can be directly derived from the slope of a $\left(\varepsilon_{\phi \psi}, \sin ^{2} \psi\right)$ plot, through the value of the elastic constants of the material.

In order to validate the assumption of an isotropic plane residual stresses state, measurements have also been performed at various $\phi$ angles. With use of the property of vanishing normal stress $\left(\sigma_{33}\right)$ at the free surface of the diffracted volume, all the components of the stress tensor and the stress-free lattice parameter can be determined (the latter is linked to $\theta_{0}$ by the Bragg relation: $2 d^{0} \sin \theta_{0}=\lambda$ ).

Figures 3 and 4 show that, for several thickness values $(\phi=0)$ and for several $\phi$ angles $\left(t^{\mathrm{f}}=1.6 \mu \mathrm{m}\right)$, the relation between $\varepsilon_{\phi \psi}$ and $\sin ^{2} \psi$ is linear; this result confirms the implicit assumption of a negligible stress gradient in the diffracted volume. For the 0.6 and $1.6 \mu \mathrm{m}$ thick coatings (A and C, Fig. 4), the whole thickness of the coatings has been investigated. For different $\phi$ angles and with the assumption of vanishing $\sigma_{33}$, all the components of the stress tensor of samples B, D, G and J have been estimated (Table 3 ).

The good agreement that can be observed

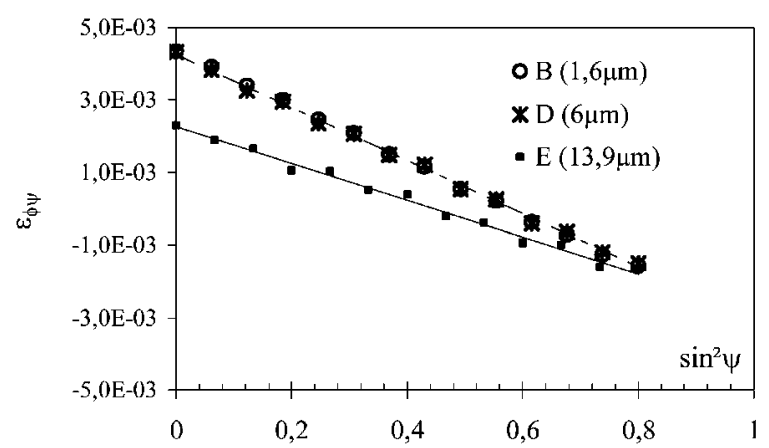

Fig. 3. $\left(\varepsilon_{\phi \psi}, \sin ^{2} \psi\right)$ plots for different thickness values of PVD coatings. 


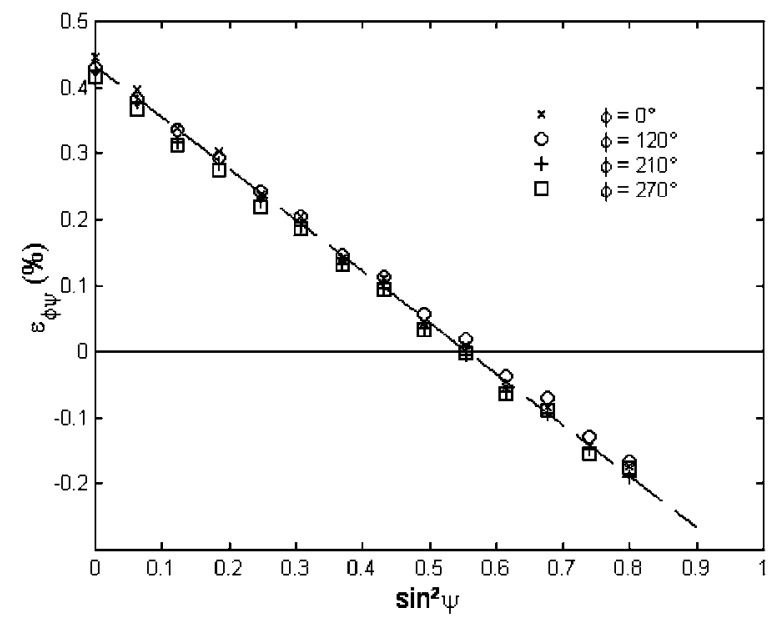

Fig. 4. $\left(\varepsilon_{\phi \psi}, \sin ^{2} \psi\right)$ plots for different angles $\phi$ for the 1.6 thick PVD W coatings.

between the results obtained from these two methods definitely validates the assumption of an isotropic plane residual stress state in the coatings. Moreover, these results are in agreement with numerical results from 3D finite element computations where the residual stresses are generated by an adequate temperature field in a bimaterial with different thermal expansion coefficients. The results are reported in Appendix A. They show that the stress gradient is negligible in the coating thickness, which validates our experimental results.

The stress-free lattice parameter has been found to be equal to $0.3168 \pm 0.0001 \mathrm{~nm}$ for samples $\mathrm{A}$ to $\mathrm{I}$ and to $0.3170 \pm 0.0002 \mathrm{~nm}$ for samples $\mathrm{J}$ and $\mathrm{K}$.
These values are a little bit larger than the bulk tungsten parameter $(0.3165 \mathrm{~nm})$, which is in agreement with previous results on PVD tungsten coatings [15-16].

\subsection{Chemical analysis}

Gergaud [10] has shown that, for identical processing conditions, the gas (argon) concentration in PVD Mo coatings increases with the development of a $\langle 111\rangle$ texture. In order to check this effect on the growth of our PVD tungsten coatings, the amount of gas (Ar) embedded in tungsten coatings has been determined by electron probe microanalysis (EPMA) with a Cameca SX-50 device. The $\mathrm{K}_{\alpha} \mathrm{X}$-ray emission of argon emitted by the sample under electron bombardment $(20 \mathrm{kV}, 100$ $\mathrm{nA}$ ) has been compared to the analysis of an $\mathrm{Ar}-$ Si standard sample containing 5.2\%wt. Ar. No gradient of the chemical composition has been observed through the coating thickness. The Ar concentration is equal to $0.2 \pm 0.1 \%$ at. for samples A to I and $0.3 \pm 0.1 \%$ at. for samples $\mathrm{J}$ and $\mathrm{K}$. This result can be related with the differences of processing conditions and with the fact that the lattice stress-free parameter $d^{0}$ is slightly larger for samples $\mathrm{J}$ and $\mathrm{K}$ than for the other ones.

\subsection{Discussion}

There is a definite correlation between the texture development and the decrease of the residual stresses level when the coating thickness is increas-

Table 3

Residual stresses of PVD W coatings determined by X-ray diffraction with the assumptions of isotropic plane residual stresses and $\sigma_{33}=0$

\begin{tabular}{|c|c|c|c|c|}
\hline Sample & Thickness $(\mu \mathrm{m})$ & $\begin{array}{l}\text { (Assumption: isotropic plane stress) } \sigma \\
\text { (MPa) }\end{array}$ & (Assumption: $\left.\sigma_{33}=0\right)(\mathrm{MPa})$ & \\
\hline B & 1.6 & $-2460 \pm 30$ & $\begin{array}{l}\sigma_{11}=-2443 \pm 60 \\
\sigma_{22}=-2507 \pm 30\end{array}$ & $\sigma_{12}=\sigma_{13}=\sigma_{23}=0 \pm 50$ \\
\hline $\mathrm{D}$ & 6 & $-2390 \pm 50$ & $\begin{array}{l}\sigma_{11}=-2374 \pm 40 \\
\sigma_{22}=-2462 \pm 45\end{array}$ & $\sigma_{12}=\sigma_{13}=\sigma_{23}=0 \pm 50$ \\
\hline G & 31 & $-1590 \pm 30$ & $\begin{array}{l}\sigma_{11}=-1602 \pm 55 \\
\sigma_{22}=-1450 \pm 55\end{array}$ & $\sigma_{12}=\sigma_{13}=\sigma_{23}=0 \pm 50$ \\
\hline $\mathrm{J}$ & 30 & - & $\begin{array}{l}\sigma_{11}=-3497 \pm 83 \\
\sigma_{22}=-3385 \pm 83\end{array}$ & $\sigma_{12}=\sigma_{13}=\sigma_{23}=0 \pm 50$ \\
\hline
\end{tabular}


ing. Dobrev [9] has proposed to correlate this texture development with the ion channelling directions in the crystal lattice. For a b.c.c lattice, these channelling directions are more and more favorable in the sequence $\langle 110\rangle,\langle 100\rangle,\langle 111\rangle$. During the impact between an energetic particle and the crystal lattice, the energy dissipation induces either a partial recrystallisation due to the "thermal spike" effect or the creation of defects due to consecutive binary collisions. Therefore, under this bombardment, the coating is growing along the more favorable channelling directions, which corresponds to a $\langle 111\rangle$ growth direction for a b.c.c lattice.

Moreover, according to Thornton and Hoffman [1], the creation of defects increases the intrinsic residual stress level. As the size of the affected region increases in the sequence $\langle 110\rangle,\langle 100\rangle$, $\langle 111\rangle$, a $\langle 111\rangle$ growth direction induces a lower density of defects and subsequently a decrease of the intrinsic residual stress level. This is in agreement with our experimental observations: when the coating thickness is increasing, a $\langle 111\rangle$ texture develops and the residual stress level decreases.

\section{Cracking behaviour}

The experimental devices used to characterize the cracking behavior of magnetron tungsten coatings are first described; we then report on the results of an investigation of the cracking mechanisms.

The substrate-coating samples are submitted to tensile tests, at a nominal $3.10^{-4} \mathrm{~s}^{-1}$ strain rate, in a Raith tension loading stage which lies inside the chamber of a scanning electron microscope (SEM). Such a device allows a more precise and realistic investigation of the cracking mechanisms under loading, of the cracking kinetics and of the substrate-coating adhesion. The tests are instrumented with an extensometer device and coupled with an acoustic emission analysis, using a piezo-electric sensor with a $20 \mathrm{kHz}$ filter and a sampling period of $100 \mathrm{~ns}$ (Fig. 5), so as to be able to determine the critical strain $( \pm 0.01 \%)$ for crack initiation and to analyse the cracking kinetics.

Several variables [17] can be used to characterise the acoustic signals $V(t)$, such as their maximum amplitude $\left(V_{\mathrm{c}}\right)$, their average amplitude $\left(V_{\mathrm{m}}\right)$ or the efficient value (i.e., the root mean square), $V_{\text {R.M.s. }}$ of the acoustic signals. The definition of the latter coefficient $V_{\text {R.M.S. }}$ is given by:

$V_{\text {R.M.S. }}(t)=\sqrt{\frac{1}{T} \int_{t}^{t+T}[V(\tau)]^{2} \mathrm{~d} \tau} \quad T=10 \mathrm{~ms}$ to a few seconds.

A shape factor, $F$, can also be used [18]: it is defined as the ratio of the maximum amplitude $\left(V_{\mathrm{c}}\right)$ to the efficient value ( $V_{\text {R.M.s. }}$ ).

The correlation between acoustic emission signals recorded during mechanical tests and crack initiation has first been investigated. In addition, four-point bending tests have been performed inside the S.E.M. chamber (Fig. 6) to allow the observation of the interface between the substrate and the coating under loading. Gold microgrids [19] of $2 \mu \mathrm{m}$ path have been deposited on the cross-section of the samples in view of a qualitative analysis of the strain field heterogeneity.

\subsection{Damage analysis}

SEM observations show a strong influence of the coating thickness on the cracking behaviour. For thinner coatings $(0.6$ and $1.6 \mu \mathrm{m})$, cracks follow the grain boundaries of the substrate, which are revealed by ion etching (Fig. 7(a)) performed inside the deposition chamber in order to clean the substrate surface and to improve the interface adhesion. Similar observations have been made on $3 \mu \mathrm{m}$ thick TiN coatings deposited on a steel substrate [20].

For thicker coatings $(6,13.9,30 \mu \mathrm{m})$, cracks are straight and perpendicular to the tensile axis (Fig. 7(b)). This mode I cracking behaviour of PVD coatings is frequently observed under bending and tensile loadings [20-21].

No debonding has been observed at the interface but strain localisation develops in the substrate at the crack tips, as revealed during a four-point bending test, by the deformation field of a gold microgrid deposited on the crack tip area of a 6 $\mu \mathrm{m}$ thick coating (Fig. 8).

The microgrid is clearly deformed close to the 


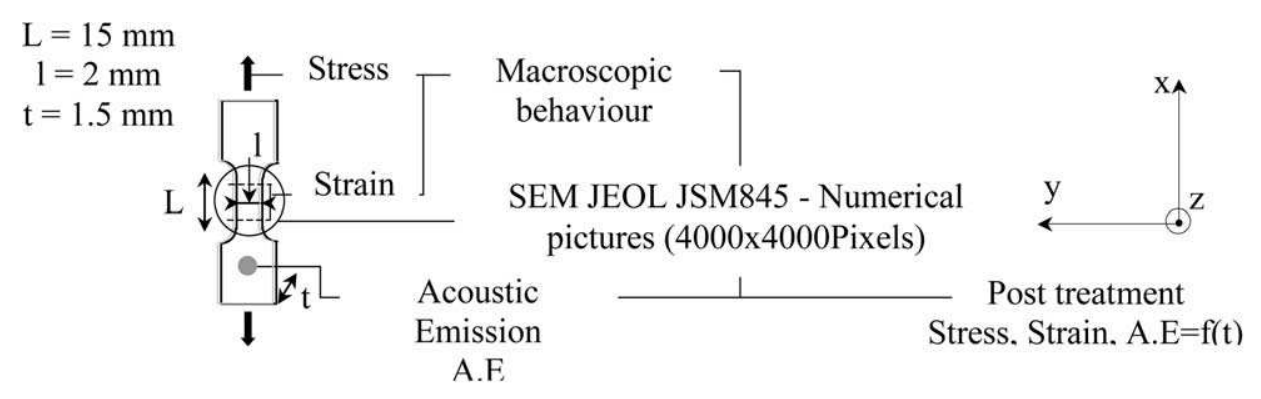

Fig. 5. Experimental tensile device used to study the cracking behaviour of coatings.

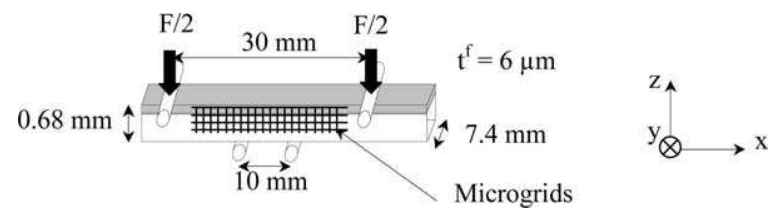

Fig. 6. Four point bending sample.

crack tip (Fig. 8) and plastic strain mechanisms appear to be active in the substrate inside deformation bands inclined by $45^{\circ}$ with respect to the substrate-coating interface. At a 10-14 $\mu \mathrm{m}$ distance of the crack, the microgrid is more homogeneously deformed and the strain level is lower than in the crack tip zone (Fig. 8).

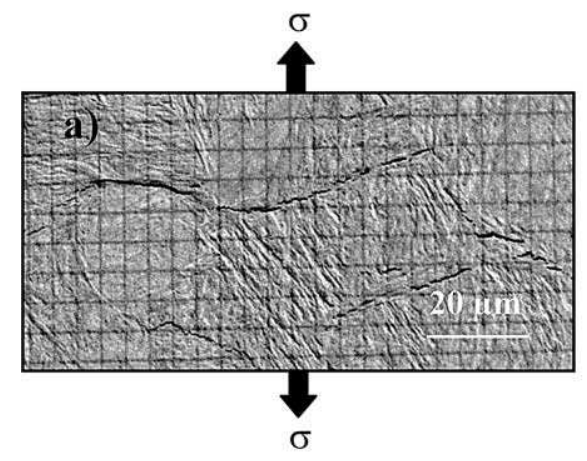

\subsection{Acoustic emission analysis}

The influence of the substrate yield stress is illustrated in Fig. 9 by the responses of a similar $13.9 \mu \mathrm{m}$ thick coating deposited either on type 1 or type 2 substrates. The black square points correspond to $(\sigma, \varepsilon)$ values associated with acoustic signals detected during the test. The acoustic signals are characterised by their $F$ ratio, which can be used as an acoustic signature of the cracks. As this ratio $F$ is found to be constant during the tensile test (Fig. 10), it can be concluded that one cracking mechanism only is active throughout the test. We can infer from this result that no interface debond-

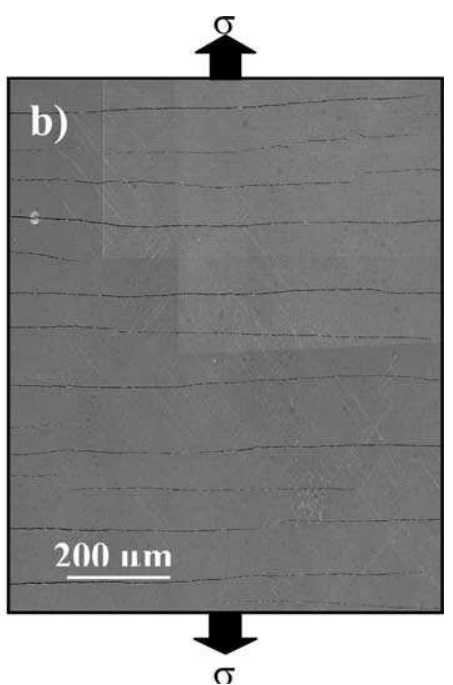

Fig. 7. Damage mechanisms observed on the coating surface (SEM images) - (a) $t_{\mathrm{f}}=0.6 \mu \mathrm{m}, \varepsilon=3 \%$, Sample B; (b) $t_{\mathrm{f}}=13.9 \mu \mathrm{m}$, $\varepsilon=5.3 \%$, Sample E, mechanisms observed for a thickness larger than $6 \mu \mathrm{m}$. 


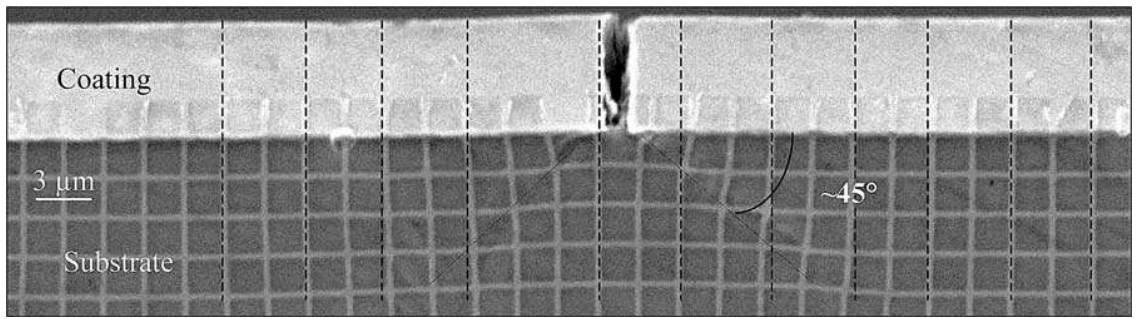

Fig. 8. Microgrid deformation close to the crack during a four-point bending test on a $6 \mu \mathrm{m}$ thick coating with a type 1 substrate.

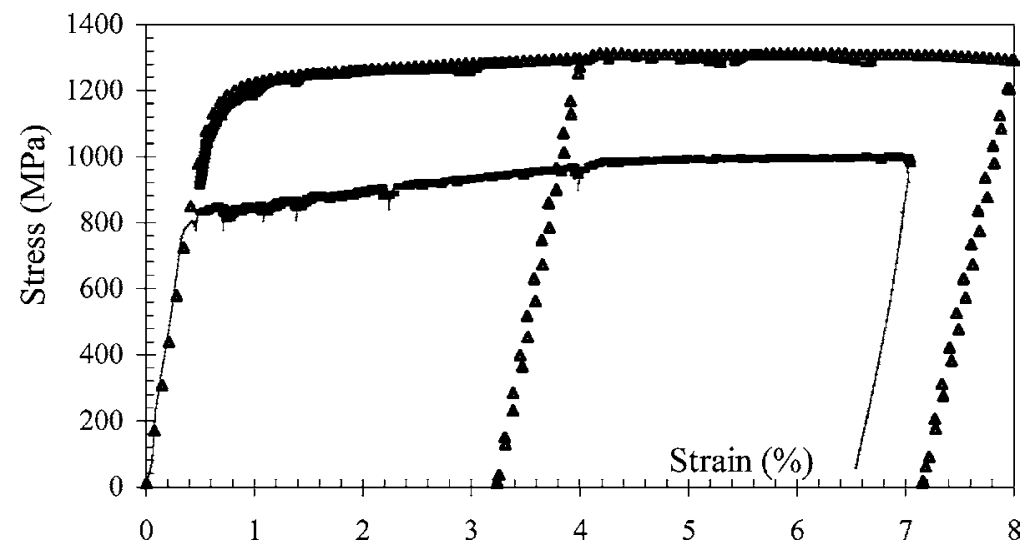

Fig. 9. Global responses of a W PVD coating sample $\left(\mathrm{E}-\mathrm{F}, t_{\mathrm{f}}=13.9 \mu \mathrm{m}\right)$ deposited on substrates 1 and 2: characteristic curve of the $\mathrm{W}$ coating/steel samples for a thickness larger than $6 \mu \mathrm{m} . \triangle: \mathrm{E}-\mathrm{W} /$ substrate $1 ;-\mathrm{F}-\mathrm{W} /$ substrate $2 ; \mathbf{\square}:(\sigma, \varepsilon)$ values associated with acoustic emission.

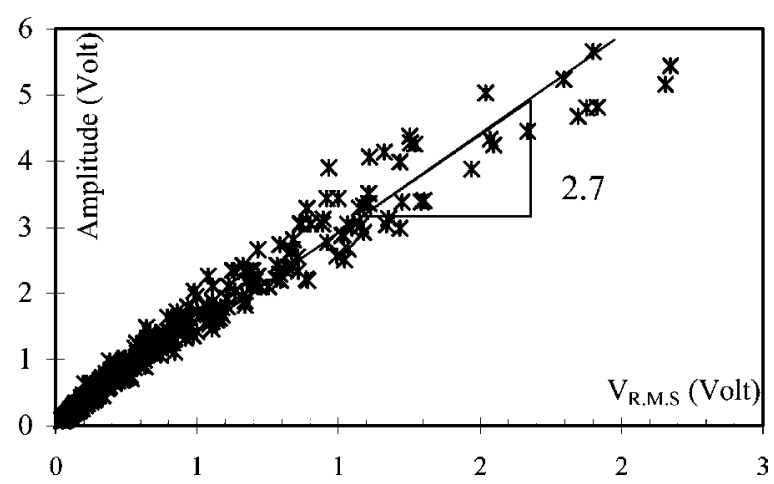

Fig. 10. Amplitude vs. $V_{\text {r.m.s. }}$ plots for a $31 \mu \mathrm{m}$ thick PVD coating. This curve is characteristic of the $\mathrm{W}$ coating/steel systems under tensile loading for a coating thickness larger than $6 \mu \mathrm{m}$. ing occurs. This conclusion is corroborated by direct SEM observations.

\subsection{Intrinsic critical cracking stress and energy release rate}

\subsubsection{Approaches}

The experimental results of the tensile tests have been reported in Table 4 for all the samples with mention of their thickness, their residual stresses and the substrate type. It can be noticed (Table 4) that the strain $\varepsilon_{x x, \mathrm{c}}^{\mathrm{s}}$ associated with cracking initiation depends on the coating thickness, so that it cannot be considered as an intrinsic damage parameter for tungsten coatings. Since it is likely to depend on the residual stress level, the associated critical stress parameter can be thought to lead to a more relevant cracking criterion.

In order to check this point, we consider a coating-substrate perfectly bound system, subjected to 
Table 4

Values of residual stresses $\left(\sigma_{\mathrm{r}}^{\mathrm{f}}\right), \varepsilon_{x x, \mathrm{c}}^{\mathrm{s}}, \sigma_{x x, \text { appl }}^{\mathrm{f}}, \sigma_{x x, \mathrm{c}}^{\mathrm{f}}$ and $G$ for different coating thickness values and substrates (the residual stresses derive from previous measurements; see Section 3.2)

\begin{tabular}{lllllll}
\hline $\begin{array}{l}\text { Substrate } \\
\sigma_{\mathrm{y}}(\mathrm{MPa})\end{array}$ & $\begin{array}{l}\text { Coating } \\
\text { thickness }(\mu \mathrm{m})\end{array}$ & $\sigma_{\mathrm{r}}^{\mathrm{f}}(\mathrm{MPa})$ & $\varepsilon_{x x, \mathrm{c}}^{\mathrm{s}}(\%)( \pm 0.01 \%)$ & $\begin{array}{l}\sigma_{x, \text { appl }}^{\mathrm{f}} \\
(\mathrm{MPa})\end{array}$ & $\sigma_{x x, \mathrm{c}}^{\mathrm{f}}(\mathrm{MPa})$ & $G\left(\mathrm{~J} / \mathrm{m}^{2}\right)$ \\
\hline A 1100 & 0.6 & -2560 & $0.88 \%>\varepsilon>0.55 \%$ & & $/$ & $/$ \\
B 1100 & 1.6 & -2460 & $0.88 \%>\varepsilon>0.55 \%$ & & $/$ & $/$ \\
C 1100 & 1.6 & -2450 & $0.68 \%>\varepsilon>0.66 \%$ & & $250-330 \pm 50$ & $/$ \\
D 1100 & 6 & -2390 & $0.66 \%$ & 2700 & $310 \pm 50$ & $3.4 \pm 1.0$ \\
E 1100 & 13.9 & -1670 & $0.47 \%$ & 1930 & $260 \pm 50$ & $5.2 \pm 1.8$ \\
G 1100 & 31.1 & -1600 & $0.46 \%$ & 1890 & $290 \pm 50$ & $15.5 \pm 4.8$ \\
H 1100 & 30.9 & -1500 & $0.43 \%$ & 1765 & $265 \pm 50$ & $12.0 \pm 4.2$ \\
J 1100 & 30.2 & -3500 & $0.92 \%$ & 3770 & $270 \pm 50$ & $12.6 \pm 4.3$ \\
F 800 & 13.9 & -1680 & $0.47 \%$ & 1930 & $250 \pm 50$ & $3.8 \pm 1.4$ \\
I 800 & 30.4 & -1550 & $0.44 \%$ & 3810 & $260 \pm 50$ & $11.3 \pm 4.1$ \\
K 800 & 30.2 & -3500 & $0.93 \%$ & $310 \pm 50$ & $17.4 \pm 5.1$ \\
\hline
\end{tabular}

uniaxial tension along the $x$ axis (Fig. 5). Both materials are assumed to be elastic, homogeneous and isotropic; the coating is submitted to an isotropic plane residual stress state. Since the coating is much thinner than the substrate, stresses can be assumed as uniform throughout the coating thickness with $\sigma_{i z}=0$. Using the continuity conditions on the displacements and tensions across the interface, we easily obtain [22]:

$$
\Rightarrow \mid \begin{aligned}
& \sigma_{x x}^{\mathrm{f}}=\frac{1-v^{\mathrm{f}} v^{\mathrm{s}}}{1-v^{\mathrm{f}_{2}}} E^{\mathrm{f}} \cdot \varepsilon_{x x}^{\mathrm{s}}+\sigma_{\mathrm{r}}^{\mathrm{f}} \\
& \sigma_{y y}^{\mathrm{f}}=\frac{v^{\mathrm{f}}-v^{\mathrm{s}}}{1-v^{\mathrm{f}}{ }^{\mathrm{f}}} \cdot \varepsilon_{x x}^{\mathrm{s}}+\sigma_{\mathrm{r}}^{\mathrm{f}}
\end{aligned},
$$

where indices $\mathrm{f}$ and $\mathrm{s}$ refer to the film and the substrate, respectively, and $\mathrm{r}$ stands for "residual". As the Poisson ratios $v^{\mathrm{f}}$ and $v^{\mathrm{s}}$ are almost equal $\left(v^{\mathrm{f}}=0.28\right.$ and $\left.v^{\mathrm{s}}=0.29\right)$, eq. (6) simplify into

$\Rightarrow \mid \sigma_{x x}^{\mathrm{f}} \approx E^{\mathrm{f}} \cdot \varepsilon_{x x}^{\mathrm{s}}+\sigma_{\mathrm{r}}^{\mathrm{f}} ; \sigma_{y y}^{\mathrm{f}} \approx \sigma_{\mathrm{r}}^{\mathrm{f}}$.

So the cracking initiation can be characterised by the critical stress $\sigma_{x x, \mathrm{f}}^{\mathrm{f}}$, associated with the critical strain $\varepsilon_{x x, \mathrm{c}}^{\mathrm{s}}$ through the relation:

$\sigma_{x x, \mathrm{c}}^{\mathrm{f}} \approx E^{\mathrm{f}} \cdot \varepsilon_{x x, \mathrm{c}}^{\mathrm{s}}+\sigma_{\mathrm{r}}^{\mathrm{f}}$

The energy release rate $G$ for the crack extension through the film can be determined for all coatingsubstrate systems from the $\mathrm{Hu}$ and Evans approach [4]. The derivation assumes an elastic behaviour of both the substrate and the coating but considers a plastic yielding zone at the crack tip in the substrate. The interface between the coating and the substrate is considered as perfect. This approach, described in [4], is based on an energy balance of a strained system when the coating is cracking. It leads to the following expression for $G$ :

$G=\frac{\left(\sigma_{x x, \mathrm{c}}^{\mathrm{f}}\right)^{2} \cdot t^{\mathrm{f}}}{E^{\mathrm{f}}}\left[\pi \cdot F\left(\frac{E^{\mathrm{s}}}{E^{\mathrm{f}}}\right)+\frac{\sigma_{x x, \mathrm{c}}^{\mathrm{f}}}{3 \cdot \tau_{y}^{\mathrm{s}}}\right]$,

where $F=\left(E^{\mathrm{s}} / E^{\mathrm{f}}\right)$ is a function of the elastic contrast [4] and $\tau_{y}^{\mathrm{s}}$ is the shear yield stress of the substrate. For our systems, $F$ is equal to 0.71 .

\subsubsection{Results}

The results corresponding to the fracture properties of tungsten coatings are presented in Table 4. Except for very thin coatings (samples A, B and C), the resulting $\sigma_{x x, \mathrm{c}}^{\mathrm{f}}$ values are found to be remarkably close to each other $\left(\sigma_{x x, \mathrm{c}}^{\mathrm{f}} \approx 275 \pm 50 \mathrm{MPa}\right)$ whatever the coating thickness and the substrate yield stress (Table 4), so that they can be considered as defining an intrinsic critical cracking stress. This value is in good agreement with previous results derived from a 3-point bending test for PVD tungsten coatings deposited on a steel substrate [7]. Nevertheless, our experimental investigation, which has been performed under homogeneous loading conditions, leads to a more accurate determination of this parameter and to a 
more conclusive proof of its intrinsic nature, especially through the independence of the critical measured stress with respect to the processing conditions. For samples A to C, whose released energy associated to cracking seems to be very low, the used acoustic sensor was not sensitive enough to detect the first cracking events.

The associated energy release rate $G$ values range from 3.4 to $17.4 \mathrm{~J} / \mathrm{m}^{2}$. They are in agreement with those determined by Harry with the same approach. A dependence of $G$ on the coating thickness can be observed since $G$ is proportional to $t^{\mathrm{f}}$ (see eq. (9)).

\subsubsection{Discussion}

A first observation can be made on the measurement uncertainty. Although the determination of the critical strain is quite precise $\left(10^{-4}\right)$, it induces a precision of the order of $18 \%$ for the critical stress and $30 \%$ for the energy release rate. Despite this fact, the order of magnitude of the obtained values for $\sigma_{x x, \mathrm{c}}^{\mathrm{f}}$ and $G$ can be considered and discussed.

Even if the brittle behaviour of tungsten coatings is qualitatively consistent with the one of the bulk material obtained from tensile loading at $20^{\circ} \mathrm{C}$ [23], there is a great difference on the level of fracture stress between our experimental results and those reported in literature. According to [24], the fracture stress is in the order of $560 \mathrm{MPa}$ for coarse tungsten and of $3900 \mathrm{MPa}$ for a $20 \mu \mathrm{m}$ diameter drawn wire. Moreover, from Farre [23], sintered tungsten has a fracture stress of $1000 \mathrm{MPa}$ and a cleavage stress of $2100 \mathrm{MPa}$. These values of intragranular fracture stress are much greater than ours: this difference suggests an intergranular fracture mechanism for our coatings. Moreover, as the cleavage stress increases with the decrease of the grain size, the intergranular fracture is all the more favourable because our coatings have a columnar microstructure with a grain size in the order of 10 to $100 \mathrm{~nm}$ (Fig. 11).

On the other side, $G$ is defined as the available energy of the crack extension through the coating per surface unit. So a crack develops if $G$ is larger than or equal to twice the surface free energy value $\left(2 \gamma_{c}\right)$. This value, which is independent of the coating thickness, is then overestimated by our experi-

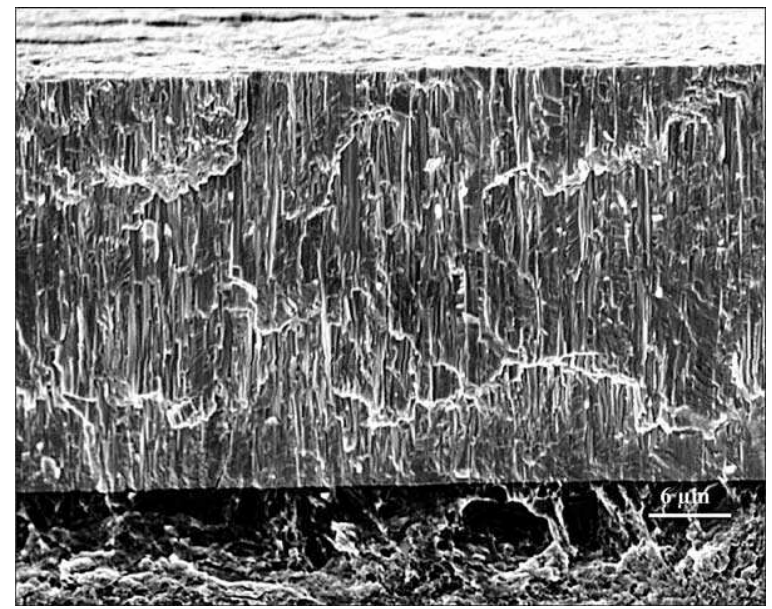

Fig. 11. SEM photograph of the columnar microstructure of $30 \mu \mathrm{m}$ thick PVD tungsten coating.

mental determination, which is concerned with the thinnest coating exhibiting mode I cracks $(6 \mu \mathrm{m})$. In the case of cleavage fracture of tungsten, Miannay [25] has shown that the theoretical fracture energy $\left(2 \gamma_{c}\right)$ is equal to $6.3,9.2,16.3 \mathrm{~J} / \mathrm{m}^{2}$ for (110), (100) and (111) planes, respectively. In addition, for b.c.c. materials, the cleavage planes are not the (110) (lowest energy) but the (100) planes. To explain this effect, Cottrell has proposed a mechanism based on the self-locking of slip systems which is responsible for strong interactions between two (110) dislocation systems and for the formation of locks: this results in an increase of the fracture energy with regard to the theoretical values [26]. Consequently, whereas our experimental values of $G$ have been overestimated, the theoretical predictions can be considered as underestimated. Nevertheless, our values are lower than the theoretical ones by a factor of 2.7 , which is certainly underestimated. Since this difference is much larger than the experimental uncertainty of our $G$ measurements (30\%), it can be concluded that the energy approach also suggests a mechanism of intergranular fracture of our PVD tungsten coatings, associated with their specific small grained columnar microstructure which is not expected in bulk tungsten specimens. This fracture mode is likely to be strongly favoured by the large specific area of grain boundaries in PVD coatings. 
Table 5

Average minimum spacing between two consecutive cracks $\left(\lambda_{\text {exp }}\right)$ for different coating thickness values and different substrates

\begin{tabular}{llll}
\hline Coating thickness $(\mu \mathrm{m})$ & Substrate yield stress $(\mathrm{MPa})$ & $\lambda_{\text {exp }}(\mu \mathrm{m})$ & Processing conditions \\
\hline 6 & 1100 & 62 & $\mathrm{~A}$ \\
14 & 1100 & 77 & $\mathrm{~A}$ \\
14 & 800 & 108 & $\mathrm{~A}$ \\
31 & 1100 & 111 & $\mathrm{~A}$ \\
30 & 800 & 140 & $\mathrm{~A}$ \\
\hline
\end{tabular}

\section{Analysis of the minimum spacing between cracks}

The crack density, defined as the number of observed mode I cracks per unit sample length, rapidly increases with strain and then slowly tends to a saturation value. This maximum crack density is known to be decreasing with the coating thickness [7], but no experimental information is available in literature on the influence of the substrate yield stress $\sigma_{\mathrm{y}}$ on this saturation density. Since the minimum average spacing between two neighbour cracks $\left(\lambda_{\text {exp }}\right)$ is inversely proportional to the maximum crack density, the $\lambda_{\exp }$ values can be usefully compared for different coating thickness values and different substrates (Table 5).

The average minimum spacing between cracks is found to increase with the coating thickness for a given substrate and to decrease with the substrate yield stress for a given coating (same thickness and residual stress level).

These effects can be analysed according to the $\mathrm{Hu}-$ Evans model [4] whose assumptions have been given in Section 4.4.1. By use of a shear lag-type treatment with a linear redistribution of the uniaxial tensile stress $\left(\sigma_{x x}^{\mathrm{f}}\right)$ in an uncracked coating portion on a distance $d$ from the crack (Fig. 12) and assuming a perfectly plastic Von Mises behaviour of the substrate at the crack tip, we obtain:

$$
\begin{gathered}
\frac{\tau_{\mathrm{y}}}{t_{\mathrm{f}}}=\frac{\sigma_{x x}^{\mathrm{f}}}{x} \text { with } \tau_{y}=\frac{\sigma_{\mathrm{y}}}{\sqrt{3}} \text { and for } x \geq d, \\
\frac{\tau_{\mathrm{y}}}{t_{\mathrm{f}}}=\frac{\sigma_{x x, \mathrm{c}}^{\mathrm{f}}}{d}
\end{gathered}
$$

where $x$ is the distance from the crack tip, $\tau_{\mathrm{y}}$ is the substrate yield shear stress and $\sigma_{x x, \mathrm{c}}^{\mathrm{f}}$ is the critical cracking stress of the coating. The minimum spacing $\lambda$ between two cracks can be bounded by $d$ (because the cracking criterion cannot be satisfied below) and $2 d$ (because this criterion is surely satisfied beyond):

$d<\lambda<2 d$.

Consequently, we get from (10) and (11):

$$
\sqrt{3}<\frac{\lambda \cdot \sigma_{\mathrm{y}}}{\sigma_{x x, \mathrm{c}}^{\mathrm{f}} \cdot t_{\mathrm{f}}}<2 \sqrt{3}
$$

So, $\lambda$ is decreasing with the substrate yield stress and increasing with the coating thickness, as observed experimentally.

The yield shear stress $\tau_{\mathrm{y}}$ of the substrates is evaluated from the Von Mises criterion $\left(\tau_{\mathrm{y}}=\right.$

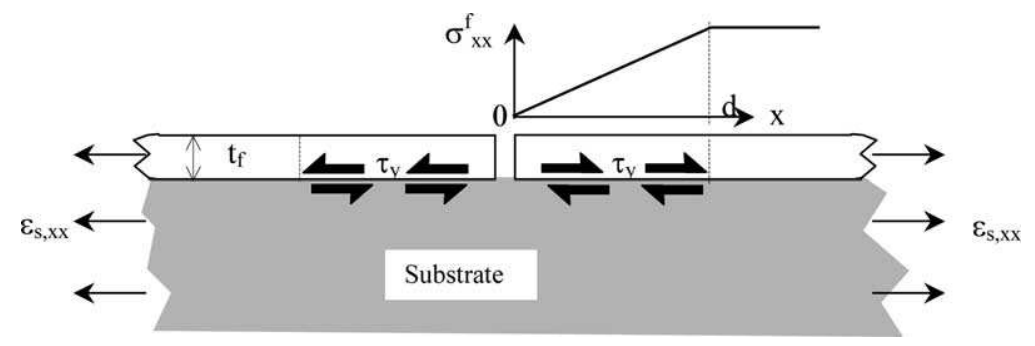

Fig. 12. Linear stress redistribution in a coating close to a crack [4]. 
$\left.\sigma_{\mathrm{y}} / \sqrt{3}\right)$ : it is equal to $635 \mathrm{MPa}$ and $460 \mathrm{MPa}$ for the substrates 1 and 2, respectively. The $\lambda$ theoretical value can be estimated from the intrinsic cracking stress and compared to the $\lambda_{\exp }$ experimental value (Table 6).

The theoretical values are significantly lower than the experimental ones. This can be considered as the consequence of the main assumption of this approach, namely the substrate strain uniformity under the coating. This assumption conflicts with what can be observed on our samples: the microgrids technique shows (Fig. 8) that the deformation field suffers a marked localisation in the substrate close to the crack tips. Consequently, although Hu-Evans's approach offers the advantage of emphasising the important role of local plasticity at the crack tips in the substrate, it strongly underestimates the strain heterogeneity induced by the cracking development and its influence on the saturation crack density value.

\section{Conclusion}

Tensile tests coupled with an acoustic emission analysis and a microextensometry technique have been used to investigate the cracking behaviour of W PVD coatings deposited on steel substrates. Taking account of the residual stresses field in the coatings, as it results from the processing conditions, an intrinsic critical cracking stress, of the order of $275 \pm 50 \mathrm{MPa}$, has been defined. This value is independent both of the coating thickness and the substrate yield stress. The low level of this intrinsic critical stress and of the associated energy release rate compared with those of bulk tungsten suggest an intergranular fracture mechanism of our
PVD coatings. This fracture mode is all the more favourable because the microstructure of coatings is small grained and columnar with an important specific area of grain boundaries.

The maximum crack density is decreasing with the coating thickness and increasing with the substrate yield stress. This effect can be explained qualitatively through a shear lag-type analysis, including plastic yielding of the substrate at the crack tips, as proposed by $\mathrm{Hu}$ and Evans [4]. Nevertheless, the development of strain localisation modes from the crack tips, deduced from the experimental analysis of the strain field in the substrate close to the interface, makes the main assumption of Hu-Evans's approach more and more inadequate as the overall strain is increasing. This strain localisation effect should be more pronounced when the coating thickness is increasing and the substrate yield stress is decreasing, which would lead to a satisfying interpretation of our experimental results.

More systematic experimental investigations are needed for the analysis of strain localisation phenomena in the substrate and for the quantitative description of their influence on the cracking kinetics of the coatings. They are in progress, in parallel with associated numerical simulations.

\section{Acknowledgements}

The authors wish to thank M. Farges (DCE/CTA) for coating elaboration, M. Le Cornec (DCE/CTA) for texture and residual stresses determination, M. Terrien (LMS) for his assistance to the acoustic emission analysis and D. Caldemaison (LMS), M. Bornert (LMS) and P. Doumalin (LMS)

Table 6

Comparison between experimental values $\left(\lambda_{\exp }\right)$ and theoretical values $(\lambda)$

\begin{tabular}{llll}
\hline Coating thickness $(\mu \mathrm{m})$ & Substrate yield stress $(\mathrm{MPa})$ & $\lambda_{\exp }(\mu \mathrm{m})$ & $\lambda(\mu \mathrm{m})$ \\
\hline 6 & 1100 & 62 & $2.6<\lambda<5.2$ \\
14 & 1100 & 77 & $6.0<\lambda<12.0$ \\
14 & 800 & 108 & $8.3<\lambda<16.6$ \\
31 & 1100 & 111 & $12.95<\lambda<25.9$ \\
30 & 800 & 140 & $17.9<\lambda<35.8$ \\
\hline
\end{tabular}


for microgrids deposition and microextensometry developments. DGA is gratefully acknowledged for financial support.

\section{Appendix A}

To generate a residual stress state in the studied structure (coating-substrate system with different thermal expansion coefficients), we can introduce an adequate temperature field without external loading. We aim at reproducing the homogeneous biaxial (plane) residual stress state in the coating determined experimentally. Due to the symmetry of the problem (Fig. 13), one quarter of the sample

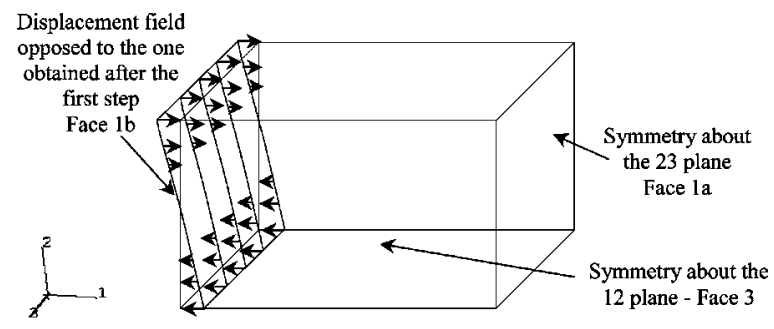

Fig. 15. Step 2, boundary conditions.

only can be studied. Faces $1 \mathrm{a}$ and 3 are symmetry planes and the other faces remain free. First, we apply a uniform temperature variation $T_{1} \rightarrow T_{0}$ (with $\left.T_{1}>T_{0}\right)$ to an elastic substrate-coating portion (Step 1).

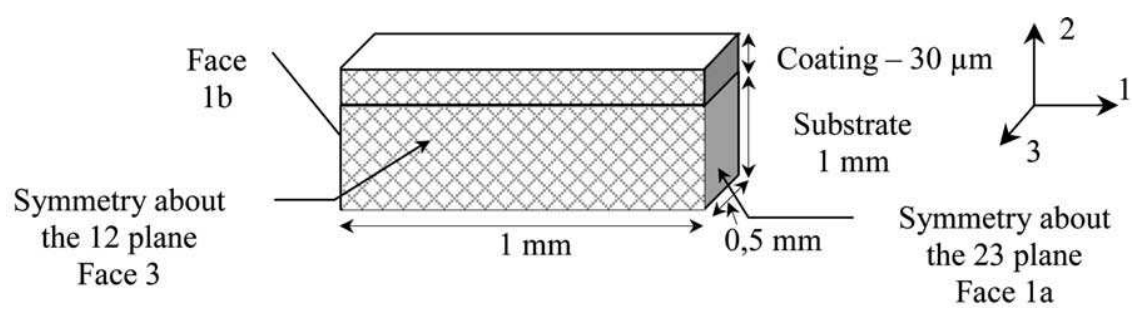

Fig. 13. Boundary conditions of the thermal-mechanical computation and geometry of the substrate-coating portion.

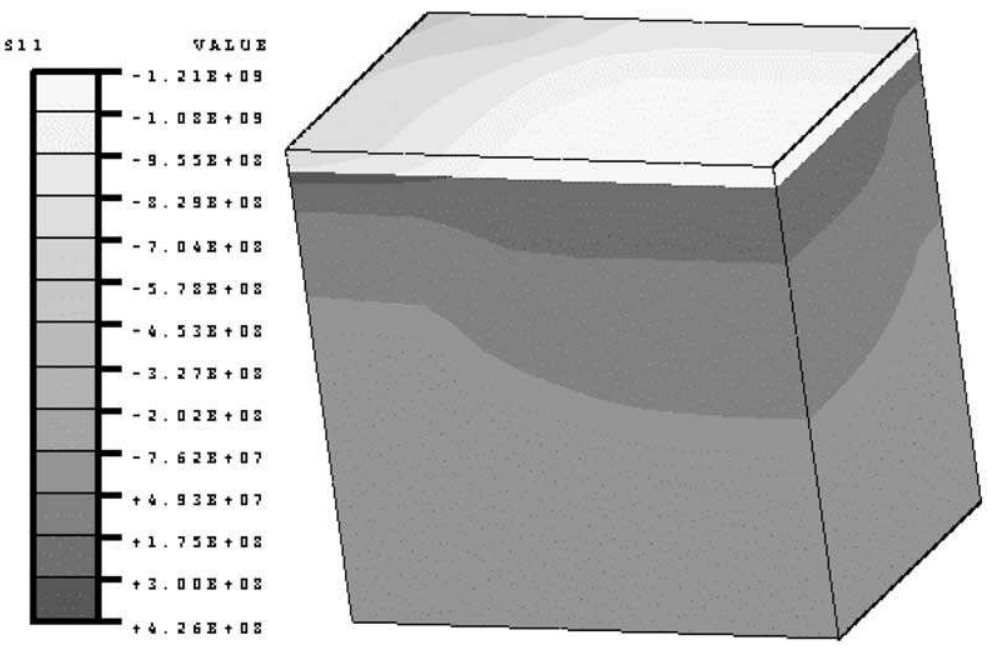

Fig. 14. Distribution of the $\sigma_{11}$ stress (Pa), step 1 (thermal-mechanical computation). 


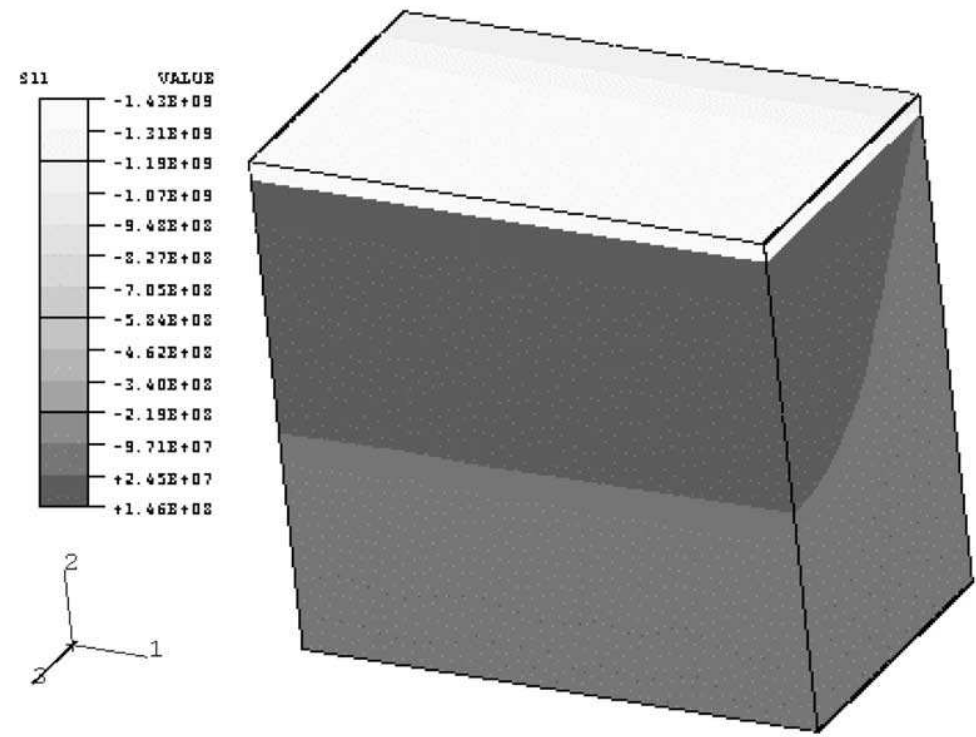

Fig. 16. Distribution of the $\sigma_{11}$ stress (Pa), step 2 .

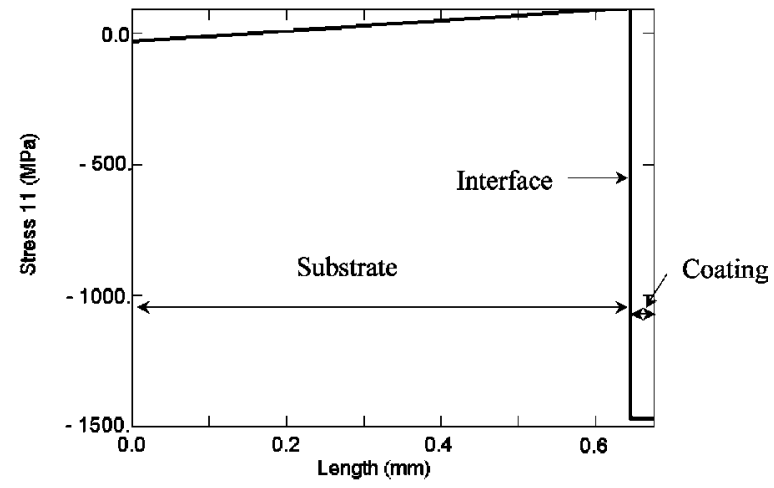

Fig. 17. $\sigma_{11}$ stress profile in the sample thickness, step 2.

This situation is not yet representative of the experimental conditions since, for a tensile specimen, the central part of the specimen is continued by coated heads. Therefore Face $1 \mathrm{~b}$ is not a free surface in the actual specimen. Consequently, we superimpose on Face $1 \mathrm{~b}$ an axial displacement field opposed to the one resulting from the computation of the first numerical Step. This displacement field is not necessarily linear and depends on the ratio of the coating thickness to the substrate thickness and on the temperature variation applied in Step 1.
So, this new boundary condition permits a realistic description of the central part of the sample without modelling the entire tensile specimen. The other faces remain free except on Face 1a and 3 (symmetry conditions) (Step 2, Fig. 15). The numerical computation was performed with the finite element software Abaqus ${ }^{\circledR}$ under the assumption of a perfect coating-substrate interface. Tungsten coating and steel substrate are considered as elastic and isotropic with temperature-independent moduli. These thermal and mechanical properties are reported in Table 1. The 3D mesh was realised through 16000 elements with 8 nodes and 8 integration points.

After the thermal computation (Step 1), the stress state of the coating is compressive and it is not homogeneous in the 1 direction. Moreover, the substrate is submitted to a stress gradient with a tensile stress state close to the interface and a compressive stress state beyond (Fig. 14).

After the Step 2 loading on Face 1b (Fig. 15), the computed stress state is almost homogeneous in the coating (Figs. 16 and 17). This stress state is representative of the experimentally measured one (Figs 16 and 18) and the stress gradient is maintained in the substrate as illustrated in Fig. 17. 


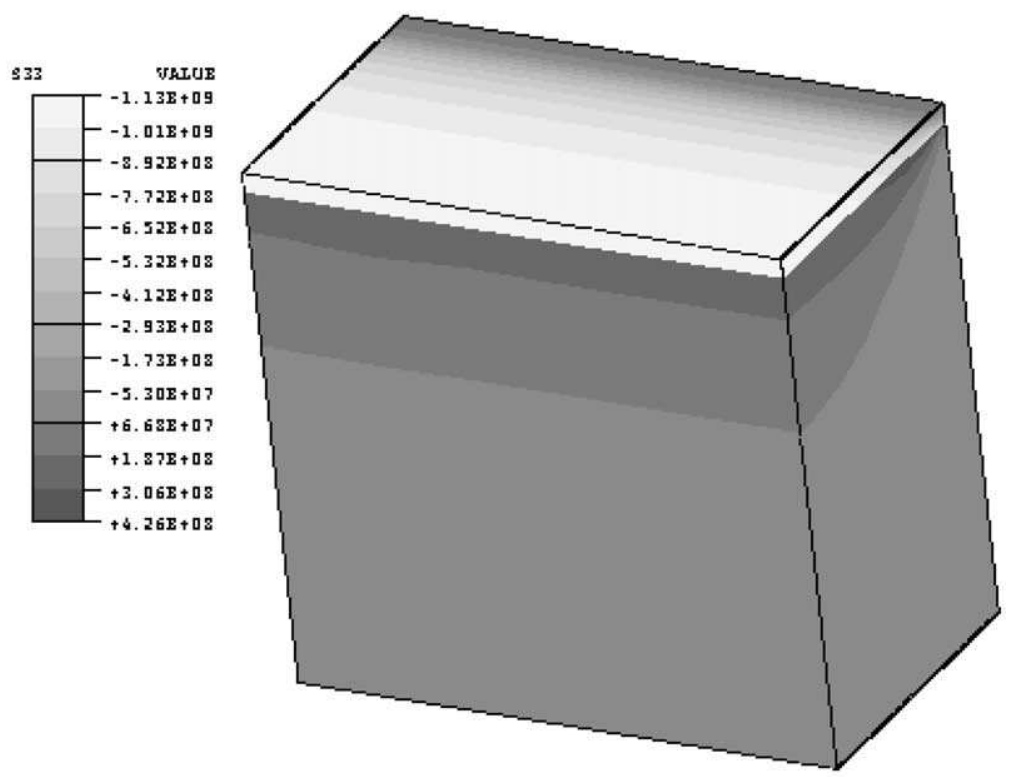

Fig. 18. Distribution of the $\sigma_{33}$ stress (Pa), step 2 .

Note that the $\sigma_{33}$ stress component does not change between Steps 1 and 2, and that the $\sigma_{33}$ stress gradient in the substrate thickness is of the same order of magnitude as the $\sigma_{11}$ stress gradient obtained after the thermal computation (Fig. 18).

\section{References}

[1] Thornton JA, Hoffman DW. Thin Solid Films; 1989;171:5.

[2] Wiklund U, Bromark M, Larson M, Hedenqvist P, Hogmark S. Surf. Coat. Technol 1997;91:57.

[3] Shojaei OR, Karimi A. Thin Solid films 1998;332:202.

[4] Hu MS, Evans AG. Acta Metall. Mater 1989;37(3):917.

[5] Patureau C, Farges G, Sainte-Catherine MC, Machet J. Surf. Coat. Technol 1998;98:1257.

[6] Rouzaud A, Barbier E, Ernoult J, Quesnel E. Thin Solid Films 1995;270:270.

[7] Harry E, Rouzaud A, Ignat M, Juliet P. Thin Solid Films 1998;332:195.

[8] Angelis C, Felder E, Farges G, Jauberteau I, Nadal M, In: Proceeding of Ann. Chim. Sci. Mat., 1998, 23, p.829.

[9] Dobrev D. Thin Solid Films 1982;92:41.

[10] Gergaud P. Ph.D. Thesis, Ecole Nationale Supérieure d'Arts et Métiers, France, 1992.

[11] Paturaud C. Ph.D. Thesis, Université de Limoges, France, 1997.
[12] Maeder G. Revue Française de Mécanique 1982;82:57.

[13] Smithells Materials Reference Book, 6th ed., Eric A. Brandes, editor.

[14] Zaouali M. Ph.D. Thesis, Ecole Nationale Supérieure d'Arts et Métiers, France, 1990

[15] Vink TJ, Walrave W, Daams JLC, Dirks AG, Somers MAJ, Van der Aker KJA. J. Appl. Phys 1993;74(2):988.

[16] Renault PO, Badawi KF, Bimbault L, Goudeau Ph, Elkhaïm E, Lauriat JP. Applied Physics Letters 1998;73(14):1952.

[17] Roget J. Techniques de l'ingénieur, R3200-1, 1999.

[18] Crépin J, Bretheau T, Caldemaison D, Ferrer F. Acta Mater 2000;48:505.

[19] Allais L, Bornert M, Bretheau M, Caldemaison D. Acta metall. Mater 1994;42:3865.

[20] Su YL, Yao SH, Wei CS, Wu CT. Thin Solid Films 1998;315:153.

[21] Scafidi P, Ignat M. J. Adhesion. Sci. Technol 1998;12(11):1219.

[22] Mezin A, Chambard JP, Lepage J, Nivoit M. Thin Solid Films 1990;185:57.

[23] Farre J, Lamaison M, Cosculluela A, Lataillade JL. J. Phys. IV France 1997;7:879.

[24] Techniques de l'ingénieur, M 2III, M570-6, 1985.

[25] Miannay D. Mécanique de la rupture. Les éditions de physique, 1995.

[26] François D, Pineau A, Zaoui A. Viscoplasticity, damage, fracture and contact mechanisms. Mechanical behaviour of materials, II. Dordrecht: Kluwer Academic; 1998. 